\title{
Partial Order in Matrix Nearrings
}

\section{Tapatee Sahoo $^{1}$. Johannes Hendrik Meyer ${ }^{2} \cdot$ Harikrishnan Panackal $^{1}$. Kedukodi Babushri Srinivas ${ }^{1}$. Kuncham Syam Prasad ${ }^{1}$}

Received: 18 June 2021 / Accepted: 6 February 2022 / Published online: 2 March 2022

(c) The Author(s) 2022

\begin{abstract}
Let $N$ be a zero-symmetric (right) nearring with identity. We introduce a partial order in the matrix nearring corresponding to the partial order (defined by Pilz in Near-rings: the theory and its applications, North Holland, Amsterdam, 1983) in N. A positive cone in a matrix nearring is defined and a characterization theorem is obtained. For a convex ideal $I$ in $N$, we prove that the corresponding ideal $I^{*}$ is convex in $M_{n}(N)$, and conversely, if $I$ is convex in $M_{n}(N)$, then $I_{*}$ is convex in $N$. Consequently, we establish an order-preserving isomorphism between the p.o. quotient matrix nearrings $M_{n}(N) / I^{*}$ and $M_{n}\left(N^{\prime}\right) /\left(I^{\prime}\right)^{*}$ where $I$ and $I^{\prime}$ are the convex ideals of p.o. nearrings $N$ and $N^{\prime}$, respectively. Finally, we prove some properties of Archimedean ordering in matrix nearrings corresponding to those in nearrings.
\end{abstract}

Keywords Nearring · Matrix nearring - Partial order

Mathematics Subject Classification 16Y30

Communicated by Ali Taherifar.

$凶 \quad$ Kuncham Syam Prasad syamprasad.k@manipal.edu

Tapatee Sahoo

tapateesahoo96@gmail.com

Johannes Hendrik Meyer

MeyerJH@ufs.ac.za

Harikrishnan Panackal

pk.harikrishnan@manipal.edu

Kedukodi Babushri Srinivas

babushrisrinivas.k@manipal.edu

1 Department of Mathematics, Manipal institute of Technology, Manipal Academy of Higher Education, Manipal, Karnataka, India

2 Department of Mathematics and Applied Mathematics, University of the Free State, Bloemfontein, South Africa 


\section{Introduction}

The notion of partial order in algebraic systems such such as groups, rings, and modules is known [6, 19]. However, the notion of partially ordered nearring (in short, p.o. nearring) was defined by Pilz $[9,10]$. Some developments in the ideal theory of partially ordered nearrings and lattice ordered nearrings were found in $[17,18]$. The purpose of this paper is to introduce and study the matrix nearrings over partial order nearrings. Matrix nearrings over arbitrary nearrings were introduced in Meldrum \& Van der Walt [11], where several results about the correspondence between the two-sided ideals in the base nearring $N$ and those in the matrix nearring $M_{n}(N)$ were proved. Later, remarkable developments in matrix nearrings over arbitrary nearrings were due to Meldrum and Meyer [12], Meyer [13, 15]. Meldrum and Meyer [12] have shown the existence of an arbitrary large lattice of ideals in the matrix nearring corresponding to an ideal in the base nearring $N$. More concepts in the ideal theory of matrix nearrings are due to [1, 3, 4]. Van der Walt [20, 21] studied the relationship between the modules over a nearring $N$, and those in modules over a matrix nearring $M_{n}(N)$. We refer to Bhavanari and Kuncham [2], Kuncham et al. [8], and Pilz [10] for fundamental notations and literature on nearrings. Further, we refer to Meldrum and Van der Walt [11], Meyer [14, 16], and Ke [7] for the comprehensive literature on matrix nearrings.

In Sect. 2, we provide the basic definitions and results in p.o. nearrings and matrix nearrings, while in Sect. 3, the positive cone is defined in the matrix nearring and obtain a characterization theorem. In Sect. 4 , the convex ideal in the matrix nearring corresponding to a convex ideal in the base nearring $N$ is introduced. Further, it is proved that if $I$ is a convex ideal of $N$, then the corresponding ideal $I^{*}$ is convex in $M_{n}(N)$; conversely, it is shown that for a convex ideal $I$ in $M_{n}(N), I_{*}$ is convex in the base nearring $N$. Consequently, an order-preserving isomorphism between the matrix nearrings $M_{n}(N) / I^{*}$ and $M_{n}\left(N^{\prime}\right) /\left(I^{\prime}\right)^{*}$ where $I$ and $I^{\prime}$ are the convex ideals of $N$ and $N^{\prime}$, respectively, is established. In the final section, we deal with the Archimedean order in a matrix nearring corresponding to the Archimedean order in $N$.

\section{Preliminaries}

An algebraic structure $(N,+, \cdot)$ is called a (right) nearring if: (i) $(N,+)$ is a group (not necessarily abelian); (ii) $(N, \cdot)$ is a semigroup; and (iii) $(a+b) c=a c+b c$ for all $a, b, c \in N$. Obviously, if $(N,+, \cdot)$ is a right nearring, then $0 a=0$ and $(-a) b=-a b$, for all $a, b \in N$, but in general $a 0 \neq 0$ for some $a \in N$. If $a 0=0$, for all $a \in N$, then $N$ is called zero-symmetric, and is denoted as $N=N_{0}$. Moreover, $n_{0}$ denotes the zero-symmetric part of the element $n \in N$. If $a a^{\prime}=a$, or $a 0=a$, for all $a, a^{\prime} \in N$, then $N$ is called a constant nearring, denoted as $N=N_{c}$. We use ' $\Leftrightarrow$ or iff' to denote 'if and only if'.

A nearring $N$ is called a partially ordered (abbr. p.o.) nearring (Pilz [10]) if $(N,+)$ is a partially ordered group under a relation ' $\leq$ ', and for any $a, b, c \in N$,

(i) $a \leq b, c \geq 0$ implies $c a \leq c b$,

(ii) $a \leq b, c \geq 0$ implies $a c \leq b c$. 
The case, if $0 \leq a$ and $a \neq 0$ is denoted as $0<a$.

If the partial order is a full order (or total order) [5], then the nearring is said to be a fully ordered (f.o.) (or a totally ordered) nearring. An element $a \in N$ is called positive if $a \geq 0$, and $N^{+}=\{a \in N \mid a \geq 0\}$, is called the positive cone. If $P$ is the positive cone of a p.o. nearring $N$, then $P$ satisfies: (i) $P \cap-P=\{0\}$, (ii) $x+P=P+x$, for all $x \in N$, (iii) $P+P \subseteq P$, (iv) $P \cdot P \subseteq P$. Conversely, if $P \subseteq N$ satisfies the above conditions, then the relation ' $\leq$ ' defined by $a \leq b \Leftrightarrow b-a \in P$ is a partial order on $N$, for which $P$ is a positive cone. Let $N, N^{\prime}$ be nearrings ordered by the positive cones $P$ and $P^{\prime}$ respectively. A map $f: N \rightarrow N^{\prime}$ is said to be an orderpreserving homomorphism (or isomorphism, denoted by $\simeq_{o}$ ) if (i) $f$ is homomorphism (or isomorphism); (ii) $f(P) \subseteq P^{\prime}$. A subset $T$ of an ordered nearring $N$ is called convex if for all $t_{1}, t_{2} \in T$, and $n \in N, t_{1} \leq n \leq t_{2}$, imply $n \in T$. A partial order on an additive group $N$ is called Archimedean, if for every pair $a, b$ in $N^{+}, n a \not \leq b$ for all $n \in \mathbb{Z}^{+}$implies $a=0$. A p.o. nearring is Archimedean, if $(N,+)$ is an Archimedean group. If $N$ is not Archimedean, then there exists a pair $(a, b)$ of $N^{2}$ with $k|a|<|b|$ for all $k \in \mathbb{N}$. In this case, we say that $a$ is small with respect to $b$, denoted by $a \ll b$. For an ordered nearring $N$, an element $n \in N$ is said to be nearly constant, if $n_{0} \ll N_{c}^{*}$ where $N_{c}^{*}=N_{c} \backslash\{0\}$.

For a zero-symmetric right nearring $N$ with identity 1 , let $N^{n}$ denote the direct sum of $n$ copies of $(N,+)$. The elements of $N^{n}$ are thought of as column vectors and written as $\left\langle r_{1}, \ldots, r_{n}\right\rangle$. The symbols $i_{j}$ and $\pi_{j}$ will denote the $i^{\text {th }}$ coordinate injection and $j^{t h}$ coordinate projection functions respectively. The nearring of $n \times n$-matrices over $N$, denoted by $M_{n}(N)$, is defined to be the subnearring of $M\left(N^{n}\right)$, generated by the set of functions $\left\{f_{i j}^{r}: N^{n} \rightarrow N^{n} \mid r \in N, 1 \leq i, j \leq n\right\}$ where $f_{i j}^{r}\left\langle r_{1}, r_{2}, \ldots, r_{n}\right\rangle:=$ $\left\langle s_{1}, s_{2}, \ldots, s_{n}\right\rangle$ with $s_{i}=r r_{j}$ and $s_{k}=0$ if $k \neq i$. The elements of $M_{n}(N)$ will be referred to as $n \times n$-matrices over $N$. The zero matrix in $M_{n}(N)$ is denoted by $\overline{\mathbf{0}}=0_{M_{n}(N)}$.

Any matrix $A$ can be represented as an expression involving only the $f_{i j}^{r}$. The length of such an expression is the number of $f_{i j}^{r}$ therein. The weight $w(A)$ of $A$ is the length of an expression of minimal length for $A$. Clearly, if $A$ is represented by an expression of length $w(A) \geq 2$, then from this expression we can find representations for $A$ as either $A=B+C$ or $A=B C$, where $w(B), w(C)<w(A)$.

\section{Positive Cone in a Matrix Nearring}

Definition 3.1 Let $N$ be a partially ordered nearring with 1 .

(i) For any $A, B \in M_{n}(N)$, we define

$$
A \leq_{n} B \Leftrightarrow \pi_{i}(A \rho) \leq \pi_{i}(B \rho) \text {, for all } \rho \in\left(N^{+}\right)^{n}, A, B \in M_{n}(N),
$$

and for all $1 \leq i \leq n$.

(ii) $M_{n}(N)$ is said to be a p.o. matrix nearring if $\leq_{n}$ defined in (i) is a partial order and satisfies the monotone properties of addition and multiplication in $M_{n}(N)$. 
Lemma 3.2 If $N$ is a p.o. nearring with 1, then the relation in Definition 3.1 on $M_{n}(N)$, is a p.o. relation and hence $M_{n}(N)$ is a p.o. matrix nearring.

Proof We show that $\leq_{n}$ is a p.o. relation on $M_{n}(N)$. Since $\pi_{i}(A \rho) \leq \pi_{i}(A \rho)$, for all $A \in M_{n}(N), \rho \in\left(N^{+}\right)^{n}, 1 \leq i \leq n$, we have $A \leq_{n} A$, so $\leq_{n}$ is reflexive. To see $\leq_{n}$ is anti-symmetric, suppose $A \leq_{n} B$, and $B \leq_{n} A$. Then, $\pi_{i}(A \rho) \leq \pi_{i}(B \rho)$, and $\pi_{i}(B \rho) \leq \pi_{i}(A \rho)$ for all $A, B \in M_{n}(N), \rho \in\left(N^{+}\right)^{n}, 1 \leq i \leq n$. Since $\leq$ is anti-symmetric on $N$, we get $\pi_{i}(A \rho)=\pi_{i}(B \rho)$ for all $\rho \in\left(N^{+}\right)^{n}, 1 \leq i \leq n$. Therefore, $A=B$. Suppose $A \leq_{n} B$, and $B \leq_{n} C$. Then, $\pi_{i}(A \rho) \leq \pi_{i}(B \rho)$, and $\pi_{i}(B \rho) \leq \pi_{i}(C \rho)$ for all $A, B, C \in M_{n}(N), \rho \in\left(N^{+}\right)^{n}, 1 \leq i \leq n$. Since $\leq$ is transitive on $N$, we get $\pi_{i}(A \rho) \leq \pi_{i}(C \rho)$ for all $\rho \in\left(N^{+}\right)^{n}, 1 \leq i \leq n$, shows that $A \leq_{n} C$. Next, to see the monotonicity in $M_{n}(N)$, assume that $A \leq_{n} B$ and $\overline{\mathbf{0}} \leq{ }_{n} C$. That is, $\pi_{i}(A \rho) \leq \pi_{i}(B \rho)$, and $\pi_{i}(0 \rho) \leq \pi_{i}(C \rho)$, for all $i \in\{1,2, \ldots, n\}$ and $\rho \in\left(N^{+}\right)^{n}$. Now,

$$
\begin{aligned}
\pi_{i}((A+C) \rho) & =\pi_{i}(A \rho+C \rho) \\
& =\pi_{i}(A \rho)+\pi_{i}(C \rho) \\
& \leq \pi_{i}(B \rho)+\pi_{i}(C \rho), \text { as } N \text { is p.o. nearring } \\
& =\pi_{i}(B \rho+C \rho) \\
& =\pi_{i}((B+C) \rho)
\end{aligned}
$$

Therefore, $A+C \leq_{n} B+C$.

Further,

$$
\begin{aligned}
\pi_{i}((A C) \rho) & =\pi_{i}(A(C \rho)) \\
& \leq \pi_{i}(B(C \rho)), \text { since } N \text { is p.o. nearring and } C \rho \in\left(N^{+}\right)^{n} \\
& =\pi_{i}((B C) \rho) .
\end{aligned}
$$

Therefore, $A C \leq_{n} B C$.

Definition 3.3 The positive cone $\mathcal{P}_{M_{n}(N)}$ (or simply $\mathcal{P}$ ) of $M_{n}(N)$ is defined as

$$
\mathcal{P}=\left\{A \in M_{n}(N) \mid A \geq_{n} \overline{\mathbf{0}}\right\}
$$

and the negative cone is,

$$
-\mathcal{P}=\left\{A \in M_{n}(N) \mid A \leq_{n} \overline{\mathbf{0}}\right\}
$$

where $\overline{\mathbf{0}}$ is the zero matrix in $M_{n}(N)$.

Now, we prove a characterization for a positive cone in $M_{n}(N)$.

Theorem 3.4 Let $N$ be a p.o. nearring, and let $M_{n}(N)$ be its p.o. matrix nearring with positive cone $\mathcal{P}$. Then, 
(1) $\mathcal{P}+\mathcal{P}=\mathcal{P}$

(2) $\mathcal{P} \cap(-\mathcal{P})=\{\overline{\mathbf{0}}\}$;

(3) $\mathcal{P} \mathcal{P} \subseteq \mathcal{P}$;

(4) $A+\mathcal{P}-A \subseteq \mathcal{P}$ for all $A \in M_{n}(N)$.

Conversely, for every subset $P$ of a matrix nearring $M_{n}(N)$ fulfilling (1)-(4), we get an ordered matrix nearring $\left(M_{n}(N), \leq_{P}\right)$, where $\leq_{P}$ is defined by $A \leq_{P} B \Leftrightarrow B-A \in$ $P$.

Proof Assume that $\mathcal{P}$ is a positive cone in $M_{n}(N)$.

(1) Let $A, B \in \mathcal{P}$. Then, $A \geq_{n} \overline{\mathbf{0}}$ and $B \geq_{n} \overline{\mathbf{0}}$. Now, $\overline{\mathbf{0}} \leq_{n} A \leq_{n} A+B$ (by monotonicity), implies $A+B \in \mathcal{P}$. Therefore, $\mathcal{P}+\mathcal{P} \subseteq \mathcal{P}$. On the other hand, if $A \in \mathcal{P}$, then $A=A+\overline{\mathbf{0}} \in \mathcal{P}+\mathcal{P}$. Therefore, $\mathcal{P} \subseteq \mathcal{P}+\mathcal{P}$.

(2) As $-\mathcal{P}=\left\{A \in M_{n}(N) \mid A \leq_{n} \overline{\mathbf{0}}\right\}$, we get $\mathcal{P} \cap-\overline{\mathcal{P}}=\{\overline{\mathbf{0}}\}$.

(3) Let $I \in M_{n}(N), A \in \mathcal{P}$. Then, since $A \geq_{n} \overline{\mathbf{0}}$ and by monotone property, $I+A \geq_{n}$ $I$, implies $I+A-I \geq_{n} \overline{\mathbf{0}}$, which shows that $I+A-I \in \mathcal{P}$.

(4) Let $A, B \in \mathcal{P}$. Then, by monotonicity in $M_{n}(N), A B \geq_{n} \overline{\mathbf{0}}$. Hence, $\mathcal{P} \mathcal{P} \subseteq \mathcal{P}$. The Converse follows from Lemma 3.2 where $P=N^{+}$, the set of positive elements in $N$.

Since every non-trivially ordered nearring is infinite by ([10], Proposition 9.127), we consider the matrix nearring to be of infinite order.

Lemma 3.5 [11] We provide some properties of matrix calculations. For all $r, s, t, r_{i}$ $(1 \leq i \leq n) \in N$, and $1 \leq i, j, k, l \leq n$,

1. $f_{i j}^{r}+f_{i j}^{s}=f_{i j}^{r+s}$;

2. $f_{i j}^{r}+f_{k l}^{s}=f_{k l}^{s}+f_{i j}^{r}$, if $i \neq k$;

3. $f_{i j}^{r} f_{k l}^{s}=\left\{\begin{array}{l}f_{i l}^{r s}, \text { if } j=k \text {; } \\ f_{i l}^{r 0}, \text { if } j \neq k .\end{array}\right.$

Example 3.6 Let $(N,+, \cdot)$ be a f.o. nearring, and $x \cdot a=\left\{\begin{array}{ll}-x, & \text { if } a<0 \\ 0, & \text { if } a=0 \\ x, & \text { if } a>0\end{array}\right.$, for all $x, a \in N$. Then, $M_{n}(N)$ is a p.o. matrix nearring, with

$$
f_{i j}^{r} \cdot f_{k l}^{s}= \begin{cases}f_{i l}^{-r}, & \text { if } s<0 \\ \overline{\mathbf{0}}, & \text { if } s=0 \\ f_{i l}^{r}, & \text { if } s>0\end{cases}
$$

for all $r, s \in N, 1 \leq i, j=k, l \leq n$.

(i) For simplicity, we verify for weight 1 . Let $a \leq b$ in $N$. Then, for any $\rho=$ $\left\langle x_{1}, x_{2}, \ldots, x_{n}\right\rangle \in\left(N^{+}\right)^{n}$, and for $A=f_{i j}^{a}, B=f_{i j}^{b}$ it follows that 


$$
\begin{aligned}
\pi_{i}(A \rho)=\pi_{i}\left(f_{i j}^{a}\left\langle x_{1}, x_{2}, \ldots, x_{n}\right\rangle\right) & =\pi_{i}\left(\langle 0,0, \ldots, \underbrace{a x_{j}}_{i^{t h}}, \ldots, 0\rangle\right) \\
& =a x_{j} \\
& \leq b x_{j} \quad\left(\text { since } a \leq b \text { and } x_{j} \geq 0\right) \\
& =\pi_{i}\left(\langle 0,0, \ldots, \underbrace{b x_{j}}_{i^{t h}}, \ldots, 0\rangle\right) \\
& =\pi_{i}\left(f_{i j}^{b}\left\langle x_{1}, x_{2}, \ldots, x_{n}\right\rangle\right) \\
& =\pi_{i}(B \rho) .
\end{aligned}
$$

Therefore, $f_{i j}^{a} \leq_{n} f_{i j}^{b}$.

(ii) Let $A=f_{i j}^{r_{1}}, B=f_{i j}^{r_{2}}, C=f_{i j}^{s} \in M_{n}(N), \overline{\mathbf{0}}<_{n} f_{i j}^{s}$ and $f_{i j}^{r_{1}} \leq_{n} f_{i j}^{r_{2}}$. Then,

$A+C=f_{i j}^{r_{1}}+f_{i j}^{s}=f_{i j}^{r_{1}+s} \leq_{n} f_{i j}^{r_{2}+s}=f_{i j}^{r_{2}}+f_{i j}^{s}=B+C$, and

$C+A=f_{i j}^{s}+f_{i j}^{r_{1}}=f_{i j}^{s+r_{1}} \leq_{n} f_{i j}^{s+r_{2}}=f_{i j}^{s}+f_{i j}^{r_{2}}=C+B$.

Therefore, ' + ' is monotone.

(iii) Let $r_{1}<0<r_{2}$ and $s>0$. Let $A=f_{i j}^{r_{1}}, B=f_{i j}^{r_{2}}, C=f_{k l}^{s} \in M_{n}(N)$. Then, $A C=f_{i j}^{r_{1}} \cdot f_{k l}^{s}=f_{i l}^{r_{1} s}=f_{i l}^{r_{1}}$ and $B C=f_{i j}^{r_{2}} \cdot f_{k l}^{s}=f_{i l}^{r_{2} s}=f_{i l}^{r_{2}}$. Since $r_{1}<r_{2}$, we have $f_{i l}^{r_{1}}<_{n} f_{i l}^{r_{2}}$ implies $A C<_{n} B C$. On the other hand, $C A=f_{k l}^{s} \cdot f_{i j}^{r_{1}}=f_{k j}^{s r_{1}}=$ $f_{k j}^{-s}$ and $C A=f_{k l}^{s} \cdot f_{i j}^{r_{2}}=f_{k j}^{s r_{2}}=f_{k j}^{s}$. Since $-s<s$, we have $f_{k j}^{-s}<_{n} f_{k j}^{s}$, which implies that $C A<_{n} C B$. Therefore, '.' is monotone.

Hence, $M_{n}(N)$ is a p.o. matrix nearring.

Remark 3.7 $M_{n}(N)$ is not f.o. in general, even if $N$ is $f . o$. For example take $N=$ $(\mathbb{Z},+, \cdot)$. Then, $N$ is f.o., that is, $a \leq b$ or $b \leq a$, for all $a, b \in N$. But $M_{n}(\mathbb{Z})$ is not f.o., for let $A=f_{12}^{1}$ and $B=f_{21}^{1}$, we have $A\langle 1,1\rangle=\langle 1,0\rangle$ and $B\langle 1,1\rangle=\langle 0,1\rangle$. Clearly, $\langle 1,0\rangle \not_{n}\langle 0,1\rangle$ and $\langle 0,1\rangle \not_{n}\langle 1,0\rangle$.

\section{Convex Ideal in a Matrix Nearring}

Definition 4.1 An ideal $I$ of $M_{n}(N)$ is said to be convex, if for any $A_{1}, A_{2} \in I$, and $B \in M_{n}(N)$ with $A_{1} \leq_{n} B \leq_{n} A_{2}$ imply $B \in I$, that is, $\pi_{i}\left(A_{1} \rho\right) \leq \pi_{i}(B \rho) \leq$ $\pi_{i}\left(A_{2} \rho\right)$, for all $\rho \in\left(N^{+}\right)^{n}, 1 \leq i \leq n$, imply $B \in I$.

Proposition 4.2 [11] If I is a two-sided ideal of $N$, then $I^{*}=\left(I^{n}: N^{n}\right)=\{A \in$ $\mathbb{M}_{n}(N) \mid A \rho \in I^{n}$ for all $\left.\rho \in N^{n}\right\}$, is a two-sided ideal of $\mathbb{M}_{n}(N)$.

Lemma 4.3 If I is a two-sided convex ideal of $N$, then $I^{*}$ is a two-sided convex ideal in $M_{n}(N)$.

Proof Let $I$ be a two-sided convex ideal of $N$. In view of Proposition 4.2, it is enough to show the convexity of $I^{*}$. Let $A_{1}, A_{2} \in I^{*}$ and $B \in M_{n}(N)$ such that $A_{1} \leq_{n}$ $B \leq_{n} A_{2}$. We prove that $B \in I^{*}$. Since $A_{1}, A_{2} \in I^{*}$, we have $A_{1} \rho \in I^{n}$ and $A_{2} \rho \in I^{n}$ for all $\rho \in\left(N^{+}\right)^{n}$. Let $A_{1} \rho=\left\langle a_{1}, \ldots, a_{n}\right\rangle \in I^{n}, B \rho=\left\langle b_{1}, \ldots, b_{n}\right\rangle$ and 
$A_{2} \rho=\left\langle a_{1}^{\prime}, \ldots, a_{n}^{\prime}\right\rangle \in I^{n}$. Then, $\pi_{i}\left\langle a_{1}, \ldots, a_{n}\right\rangle \leq \pi_{i}\left\langle b_{1}, \ldots, b_{n}\right\rangle \leq \pi_{i}\left\langle a_{1}^{\prime}, \ldots, a_{n}^{\prime}\right\rangle$, implies $a_{i} \leq b_{i} \leq a_{i}^{\prime}$, for all $i \in\{1,2, \ldots, n\}$. Since $a_{i}, a_{i}^{\prime} \in I$ and $I$ is convex, we get $b_{i} \in I$, for all $i \in\{1,2, \ldots, n\}$. Therefore, $B \rho \in I^{n}$, implies $B \in I^{*}$. Hence, $I^{*}$ is convex.

Example 4.4 Consider the nearring $N=\mathbb{Z}_{2} \times \mathbb{Z}$ (where $\mathbb{Z}$ is the set of all integers), operations are defined component wise. Define ' $\leq$ ' on $N$ as $(a, b) \leq(c, d) \Leftrightarrow(a=$ $c$ and $b<d)$ or $(a<c, b<d)$. Then, ' $\leq$ ' is a partial order on $N$, and hence $N$ is a p.o. nearring. The ideals are $I_{1}=\{0\} \times \mathbb{Z}, I_{2}=\mathbb{Z}_{2} \times\{0\}$. Clearly $I_{1}$ is convex in $N$. Now, write the matrix nearring

$$
M_{n}\left(\mathbb{Z}_{2} \times \mathbb{Z}\right)=\left\langle\left\{f_{i j}^{(a, b)} \mid(a, b) \in \mathbb{Z}_{2} \times \mathbb{Z}, 1 \leq i, j \leq n\right\}\right\rangle
$$

Then, $I_{1}^{*}=\left\langle\left\{f_{i j}^{(a, b)} \mid(a, b) \in I_{1}, 1 \leq i, j \leq n\right\}\right\rangle$, is a convex ideal in $M_{n}\left(\mathbb{Z}_{2} \times \mathbb{Z}\right)$.

Theorem 4.5 [12] For an ideal $I$ of $N, I^{+}=\left\langle\left\{f_{i j}^{a} \mid a \in I, 1 \leq i, j \leq n\right\}\right\rangle$ is an ideal of $M_{n}(N)$.

Proposition 4.6 If I is a two-sided convex ideal of $\mathrm{N}$, then $\mathrm{I}^{+}$is a two-sided convex ideal in $M_{n}(N)$.

Proof Let $I$ be a convex ideal of $N$. Then, by Theorem 4.5, $I^{+}$is an ideal of $M_{n}(N)$. To show, $I^{+}$is convex in $M_{n}(N)$, let $f_{i j}^{a_{1}}, f_{i j}^{a_{2}} \in I^{+}$and $f_{i j}^{b} \in M_{n}(N)$ such that $f_{i j}^{a_{1}} \leq_{n} f_{i j}^{b} \leq_{n} f_{i j}^{a_{2}}$, for some $a_{1}, a_{2}, b \in N, 1 \leq i, j \leq n$. Then, $\pi_{i}\left(f_{i j}^{a_{1}} \rho\right) \leq$ $\pi_{i}\left(f_{i j}^{b} \rho\right) \leq \pi_{i}\left(f_{i j}^{a_{2}} \rho\right)$ for all $\rho \in\left(N^{+}\right)^{n}$. In particular, $\pi_{i}\left(f_{i j}^{a_{1}}\langle 1,1, \ldots, 1\rangle\right) \leq$ $\pi_{i}\left(f_{i j}^{b}\langle 1,1, \ldots, 1\rangle\right) \leq \pi_{i}\left(f_{i j}^{a_{2}}\langle 1,1, \ldots, 1\rangle\right)$, where $\langle 1,1, \ldots, 1\rangle \in\left(N^{+}\right)^{n}$. This implies, $\pi_{i}\left(\langle 0,0, \ldots, \underbrace{a_{1}}_{\mathrm{i}^{\text {th }}}, \ldots, 0\rangle\right) \leq \pi_{i}\left(\langle 0,0, \ldots, \underbrace{b}_{\mathrm{i}^{\text {th }}}, \ldots, 0\rangle\right) \leq \pi_{i}(\langle 0,0, \ldots$, $\underbrace{a_{2}}_{\text {ith }^{\text {th }}}, \ldots, 0\rangle)$, which shows that $a_{1} \leq b \leq a_{2}$. Since $f_{i j}^{a_{1}}, f_{i j}^{a_{2}} \in I^{+}$, it follows that $a_{1}, a_{2} \in I$. Now, since $I$ is convex, we have $b \in I$. Therefore, $f_{i j}^{b} \in I^{+}$, which proves that $I^{+}$is convex in $M_{n}(N)$.

Proposition 4.7 [11] If $I$ is a two-sided ideal of $M_{n}(N)$, then $I_{*}=\{x \in N \mid x \in$ $\operatorname{Im}\left(\pi_{j} A\right)$ for some $A \in I$ and $\left.j, 1 \leq j \leq n\right\}$ is a two-sided ideal $N$.

Corollary 4.8 [11] If I is a two-sided ideal of $M_{n}(N)$, then $a \in I_{*}$ iff $f_{i j}^{a} \in I$, for all $1 \leq i, j \leq n$.

Lemma 4.9 If I is a two-sided convex ideal of $M_{n}(N)$ then $I_{*}$ is a two-sided convex ideal in $N$.

Proof Let $I$ be a convex ideal of $M_{n}(N)$. Then, by Proposition 4.7, $I_{*}$ is an ideal of $N$. To prove $I_{*}$ is convex in $N$, let $a, b \in I_{*}$ and $r \in N$ such that $a \leq r \leq b$. 
Then, by Corollary 4.8, $f_{i j}^{a}, f_{i j}^{b} \in I$. Now, we show that $f_{i j}^{a} \leq_{n} f_{i j}^{r} \leq_{n} f_{i j}^{b}$. Let $\rho=\left\langle x_{1}, \ldots, x_{n}\right\rangle \in\left(N^{+}\right)^{n}$,

$$
\begin{aligned}
\pi_{i}\left(f_{i j}^{a} \rho\right) & =\pi_{i}\left(f_{i j}^{a}\left\langle x_{1}, x_{2}, \ldots, x_{n}\right\rangle\right) \\
& =\pi_{i}\left(\langle 0,0, \ldots, \underbrace{a x_{j}}_{i^{t h}}, \ldots, 0\rangle\right) \\
& \left.\leq \pi_{i}\left(\langle 0,0, \ldots, \underbrace{r x_{j}}_{i^{t h}}, \ldots, 0\rangle\right) \text { (by monotonicity, as } a \leq r, x_{j} \geq 0\right) \\
& =\pi_{i}\left(f_{i j}^{r}\left\langle x_{1}, x_{2}, \ldots, x_{n}\right\rangle\right) \\
& =\pi_{i}\left(f_{i j}^{r} \rho\right)
\end{aligned}
$$

implies that $f_{i j}^{a} \leq_{n} f_{i j}^{r}$. Other way,

$$
\begin{aligned}
\pi_{i}\left(f_{i j}^{r} \rho\right) & =\pi_{i}\left(f_{i j}^{r}\left\langle x_{1}, x_{2}, \ldots, x_{n}\right\rangle\right) \\
& =\pi_{i}\left(\langle 0,0, \ldots, \underbrace{r x_{j}}_{i^{t h}}, \ldots, 0\rangle\right) \\
& \leq \pi_{i}\left(\langle 0,0, \ldots, \underbrace{b x_{j}}_{i^{t h}}, \ldots, 0\rangle\right)\left(\text { by monotonicity, as } r \leq b, x_{j} \geq 0\right) \\
& =\pi_{i}\left(f_{i j}^{b}\left\langle x_{1}, x_{2}, \ldots, x_{n}\right\rangle\right) \\
& =\pi_{i}\left(f_{i j}^{b} \rho\right)
\end{aligned}
$$

implies that $f_{i j}^{r} \leq_{n} f_{i j}^{b}$. Therefore, $f_{i j}^{a} \leq_{n} f_{i j}^{r} \leq_{n} f_{i j}^{b}$. Since $I$ is convex in $M_{n}(N)$, we get $f_{i j}^{r} \in I$ and by Corollary 4.8, $r \in I_{*}$, showing that $I_{*}$ is convex in $N$.

Definition 4.10 [22] An $n \times n$-matrix $A$ over a nearring $N$ is said to be $\sigma$-generated if it can be expressed as a sum of elements $f_{i j}^{r}, r \in N, 1 \leq i, j \leq n . M_{n}(N)$ is said to be $\sigma$-generated if every $n \times n$-matrix $A$ over $N$ is $\sigma$-generated. $N$ is said to be $\sigma$-generated if $M_{n}(N)$ is $\sigma$-generated for every $n \geq 1$.

Let $E_{n}(N)$ be set of matrix expressions. The matrix represented by $U \in E_{n}(N)$ is denoted by $\mu(U)$. The depth of an expression is a unique number $d(U), U \in$ $E_{n}(N)$, assigned as follows: (i) $d\left(f_{i j}^{r}\right)=0$, (ii) $d(U+V)=\max (d(U), d(V))$, (iii) $d(U V)=d(U)+d(V)+1$. Let $N$ and $N^{\prime}$ be two nearrings and $h: N \rightarrow N^{\prime}$, $h(r)=r^{\prime}$ be a nearring epimorphism. Consider the mapping $\theta: E_{n}(N) \rightarrow E_{n}\left(N^{\prime}\right)$, where $\theta(E)=E^{\prime}$ is the expression derived from $E \in E_{n}(N)$ by replacing every $f_{i j}^{r}$ in $E$ by $f_{i j}^{r^{\prime}}$. Then, $\theta$ is onto and $\theta(E+F)=\theta(E)+\theta(F), \theta(E F)=\theta(E) \theta(F)$ for all $E, F \in E_{n}(N)$. We define $\psi: M_{n}(N) \rightarrow M_{n}\left(N^{\prime}\right)$, by $\psi(A)=\mu(\theta(E))$, where $A=\mu(E) . M_{n}(N)$ is $\sigma$-generated if every $n \times n$-matrix $A$ over $N$ has an expression $U \in E_{n}(N)$ such that $\mu(U)=A$ and $d(U)=0$.

Lemma 4.11 Let $N$ and $N^{\prime}$ be p.o. $\sigma$-generated nearrings. If $h: N \rightarrow N^{\prime}$ is an order-preserving map, then the map $\psi: M_{n}(N) \rightarrow M_{n}\left(N^{\prime}\right)$ is also order-preserving. 
Proof Let $M_{n}(N)$ and $M_{n}\left(N^{\prime}\right)$ be two p.o. matrix nearrings with positive cones $\mathcal{P}$ and $\mathcal{P}^{\prime}$ respectively. Define $\psi: M_{n}(N) \rightarrow M_{n}\left(N^{\prime}\right)$ by $\psi(I)=\mu\left(E^{\prime}\right)$ where $I=\mu(E)$ as in [22]. To show $\psi(\mathcal{P}) \subseteq \mathcal{P}^{\prime}$, let $A \in \psi(\mathcal{P})$. Then, $A=\psi(B)$, for some $B \in \mathcal{P}$. That is, $A=\psi(B)=\mu\left(E_{1}^{\prime}\right)$, where $B=\mu\left(E_{1}\right)$. Now, we apply induction on $d\left(E_{1}\right)$.

Case-(i): $d\left(E_{1}\right)=0$. Since $N$ is $\sigma$-generated, by definition, $M_{n}(N)$ is $\sigma$-generated, for all $n \geq 1$. Hence, $B$ has an expression $E_{1} \in E_{n}(N)$ such that $B=\mu\left(E_{1}\right)$ and $d\left(E_{1}\right)=0$. Let $E_{1}=f_{i j}^{r}$, where $r \in N^{+}$. Now, $A=\mu\left(E_{1}^{\prime}\right)=\mu\left(f_{i j}^{r^{\prime}}\right) \in \mathcal{P}$, where $r^{\prime} \in\left(N^{\prime}\right)^{+}$. Hence, $\psi(\mathcal{P}) \subseteq \mathcal{P}^{\prime}$. Assume that the result is true for $d\left(E_{1}\right)<t$, for all $E_{1} \in E_{n}(N)$.

Case-(ii): $d\left(E_{1}\right)=t>0$. Then, $E_{1}=U+V$ or $E_{1}=U V$, where $U, V \in E_{n}(N)$ and $d(U), d(V)<t$.

Subcase-(i): $E_{1}=U+V$. Then, $A=\psi(B)=\mu\left(E_{1}^{\prime}\right)=\mu\left(U^{\prime}+V^{\prime}\right)=\mu\left(U^{\prime}\right)+$ $\mu\left(V^{\prime}\right) \in \mathcal{P}^{\prime}+\mathcal{P}^{\prime}=\mathcal{P}^{\prime}$, by Theorem 3.4(1). Therefore, $\psi(\mathcal{P}) \subseteq \mathcal{P}^{\prime}$.

Subcase-(ii): $E_{1}=U V$. Then, $A=\psi(B)=\mu\left(E_{1}^{\prime}\right)=\mu\left(U^{\prime} V^{\prime}\right)=\mu\left(U^{\prime}\right) \mu\left(V^{\prime}\right) \in$ $\mathcal{P}^{\prime} \mathcal{P}^{\prime} \subseteq \mathcal{P}^{\prime}$, by Theorem 3.4(3). Therefore, $\psi(\mathcal{P}) \subseteq \mathcal{P}^{\prime}$.

Theorem 4.12 Let $h: N \rightarrow N^{\prime}$ be an order-preserving homomorphism, where $N$ and $N^{\prime}$ are $\sigma$-generated p.o. nearrings, such that ker $h=I$ is convex. Then, $I^{*}$ is the kernel of the order-preserving homomorphism $\psi: M_{n}(N) \rightarrow M_{n}\left(N^{\prime}\right)$, which is convex.

Proof Since $I$ is a convex ideal of $N$, by Lemma 4.3, we have $I^{*}$ is convex ideal in $M_{n}(N)$. Also, since $h$ is an order-preserving homomorphism, by Lemma 4.11, $\psi$ is order-preserving homomorphism. It remains to show that $I^{*}=$ ker $\psi$. Now,

$$
\begin{aligned}
A \in \operatorname{ker} \psi & \Leftrightarrow \psi(A)=(\overline{\mathbf{0}})^{\prime} \\
& \Leftrightarrow \psi(A) \rho^{\prime}=(\overline{\mathbf{0}})^{\prime}, \text { for all } \rho^{\prime} \in\left(N^{\prime}\right)^{n} \\
& \Leftrightarrow \mu\left(E^{\prime}\right) \rho^{\prime}=(\overline{\mathbf{0}})^{\prime} \\
& \Leftrightarrow \pi_{i} \mu\left(E^{\prime}\right) \rho^{\prime}=0_{N^{\prime}} \\
& \Leftrightarrow h \pi_{i} \mu(E) \rho=0_{N}, \text { by }([22], \text { Lemma 3.1) } \\
& \Leftrightarrow \pi_{i} \mu(E) \rho \in k e r h=I \\
& \Leftrightarrow \pi_{i} \mu(E) \rho \in I, \text { for all } i, 1 \leq i \leq n \\
& \Leftrightarrow \mu(E) \rho \in I^{n} \\
& \Leftrightarrow A \rho \in I^{n}, \text { for all } \rho \in N^{n} \\
& \Leftrightarrow A \in I^{*} .
\end{aligned}
$$

Therefore, $I^{*}=$ ker $\psi$.

Proposition 4.13 Let I be a convex ideal of a p.o. nearring $N$. Then, for any $A, B \in$ $M_{n}(N), A+I^{*} \leq_{n}^{*} B+I^{*}$ if $A \leq_{n} B+C$, for some $C \in I^{*}$.

Proof We show $\leq_{n}^{*}$ is well defined. Let $A+I^{*}, B+I^{*} \in M_{n}(N) / I^{*}$ such that $A+I^{*} \leq_{n}^{*} B+I^{*}$. Let $A+I^{*}=A_{1}+I^{*}$ and $B+I^{*}=B_{1}+I^{*}$. Then, we note that $A-A_{1} \in I^{*}$ and $B-B_{1} \in I^{*}$, implies $\left(A-A_{1}\right) \rho \in I^{n}$ and $\left(B-B_{1}\right) \rho \in I^{n}$ 
for all $\rho \in N^{n}$. Therefore, $\left(A-A_{1}\right) \rho=C_{1} \rho$ and $\left(B-B_{1}\right) \rho=C_{2} \rho$ for some $C_{1}, C_{2} \in M_{n}(N)$. That is, $A \rho-A_{1} \rho=C_{1} \rho$ and $B \rho-B_{1} \rho=C_{2} \rho$, which implies $A \rho=C_{1} \rho+A_{1} \rho$ and $B \rho=C_{2} \rho+B_{1} \rho$. Now,

$$
\begin{aligned}
\pi_{i}\left(C_{1} \rho+A_{1} \rho\right) & =\pi_{i}(A \rho) \\
& \leq \pi_{i}((B+C) \rho) \\
& =\pi_{i}(B \rho+C \rho) \\
& =\pi_{i}\left(C_{2} \rho+B_{1} \rho+C \rho\right) .
\end{aligned}
$$

Thus, $\pi_{i}\left(C_{1} \rho\right)+\pi_{i}\left(A_{1} \rho\right) \leq \pi_{i}\left(C_{2} \rho\right)+\pi_{i}\left(B_{1} \rho\right)+\pi_{i}(C \rho)$, implies

$$
\begin{aligned}
\pi_{i}\left(A_{1} \rho\right) & \leq-\pi_{i}\left(C_{1} \rho\right)+\pi_{i}\left(C_{2} \rho\right)+\pi_{i}\left(B_{1} \rho\right)+\pi_{i}(C \rho) \\
& =\pi_{i}(D \rho)+\pi_{i}\left(B_{1} \rho\right)+\pi_{i}(C \rho), \text { where } \pi_{i}(D \rho) \\
& =-\pi_{i}\left(C_{1} \rho\right)+\pi_{i}\left(C_{2} \rho\right) \in I \\
& =\pi_{i}\left(B_{1} \rho\right)+\pi_{i}(E \rho), \text { where } \pi_{i}(E \rho) \in I \\
& =\pi_{i}\left(B_{1} \rho+E \rho\right) \\
& =\pi_{i}\left(\left(B_{1}+E\right) \rho\right)
\end{aligned}
$$

for all $\rho \in\left(N^{+}\right)^{n}$, for all $i, 1 \leq i \leq n$.

This shows that $A_{1} \leq{ }_{n} B_{1}+E$, where $E \in I^{*}$. Hence, $A_{1}+I^{*} \leq_{n}^{*} B_{1}+I^{*}$.

Clearly, since $\leq_{n}$ is a partial order relation, we have $\leq_{n}^{*}$ is also a partial order relation. Now, we show the monotonicity.

(i) Let $A+I^{*}, B+I^{*} \in M_{n}(N) / I^{*}$ such that $A+I^{*} \leq_{n}^{*} B+I^{*}$. Then, $A \leq B+D$, for some $D \in I^{*}$, implies $\pi_{i}(A \rho) \leq \pi_{i}((B+D) \rho)=\pi_{i}(B \rho)+\pi_{i}(D \rho)$, for all $\rho \in\left(N^{+}\right)^{n}$, for all $i$. Now, by monotonicity in $N, \pi_{i}(A \rho)+\pi_{i}(C \rho) \leq \pi_{i}(B \rho)+$ $\pi_{i}(D \rho)+\pi_{i}(C \rho)$, for some $\pi_{i}(C \rho) \in N$. Then,

$$
\begin{aligned}
\pi_{i}((A+C) \rho) & =\pi_{i}(A \rho)+\pi_{i}(C \rho) \\
& \leq \pi_{i}(B \rho)+\pi_{i}(D \rho)+\pi_{i}(C \rho) \\
& =\pi_{i}(B \rho)+\pi_{i}(C \rho)+\pi_{i}\left(D_{1} \rho\right), \text { where } \pi_{i}\left(D_{1} \rho\right) \in I \\
& =\pi_{i}((B+C) \rho)+\pi_{i}\left(D_{1} \rho\right),
\end{aligned}
$$

for all $i, 1 \leq i \leq n$.

This shows that $A+C \leq_{n}(B+C)+D_{1}$, where $D_{1} \in I^{*}$. Hence $(A+C)+I^{*} \leq_{n}^{*}$ $(B+C)+I^{*}$. Similarly, $(C+A)+I^{*} \leq_{n}^{*}(C+B)+I^{*}$.

(ii) Let $A+I^{*}, B+I^{*} \in M_{n}(N) / I^{*}$ such that $A+I^{*} \leq_{n}^{*} B+I^{*}$. Then, $A \leq B+D$, for some $D \in I^{*}$, implies $\pi_{i}(A \rho) \leq \pi_{i}((B+D) \rho)=\pi_{i}(B \rho)+\pi_{i}(D \rho)$, for all $\rho \in\left(N^{+}\right)^{n}$, for all $i$. Now, by monotonicity in $N, \pi_{i}(A \rho) \pi_{i}(C \rho) \leq$ $\left(\pi_{i}(B \rho)+\pi_{i}(D \rho)\right) \pi_{i}(C \rho)$, for some $\pi_{i}(C \rho) \in N$. Then,

$$
\begin{aligned}
\pi_{i}((A C) \rho)=\pi_{i}(A \rho) \pi_{i}(C \rho) & \leq \pi_{i}(B \rho) \pi_{i}(C \rho)+\pi_{i}(D \rho) \pi_{i}(C \rho) \\
& =\pi_{i}((B C) \rho)+\pi_{i}((D C) \rho) \\
& =\pi_{i}((B C+D C) \rho)
\end{aligned}
$$


for all $i, 1 \leq i \leq n$.

This shows that $A C \leq_{n} B C+D C$, where $D C \in I^{*}$, as $I^{*}$ is a right ideal of $N$. Hence $(A C)+I^{*} \leq_{n}^{*}(B C)+I^{*}$. Similarly, $(C A)+I^{*} \leq_{n}^{*}(C B)+I^{*}$. Therefore, $M_{n}(N) / I^{*}$ is a p.o. matrix nearring.

Lemma 4.14 [20] If $A$ is an ideal of $N$, then $M_{n}(N / A) \simeq M_{n}(N) / A^{*}$.

Theorem 4.15 Let $N, N^{\prime}$ be p.o. $\sigma$-generated nearrings and let $h: N \rightarrow N^{\prime}$ be an order-preserving epimorphism with positive cones $P$ and $P^{\prime}$ respectively such that for every convex ideal $I^{\prime}$ of $N^{\prime}, h^{-1}\left(I^{\prime}\right)=I$ is convex, with $N / I \simeq_{0} N^{\prime} / I^{\prime}$. Then, there exists an order-preserving epimorphism $\phi: M_{n}(N) \rightarrow M_{n}\left(N^{\prime}\right)$, such that if $\left(I^{\prime}\right)^{*}$ is convex in $M_{n}\left(N^{\prime}\right)$, then $\phi^{-1}\left(\left(I^{\prime}\right)^{*}\right)=I^{*}$ is convex in $M_{n}(N)$, and $M_{n}(N) / I^{*} \simeq 。 M_{n}\left(N^{\prime}\right) /\left(I^{\prime}\right)^{*}$.

Proof Define $\phi: M_{n}(N) \rightarrow M_{n}\left(N^{\prime}\right)$ by $\phi(A)=A^{\prime}$. Let $\left(I^{\prime}\right)^{*}$ be a convex ideal of $M_{n}\left(N^{\prime}\right)$. To show $I^{*}$ is convex in $M_{n}(N)$, let $A, B \in I^{*}$ and $C \in M_{n}(N)$ such that $A \leq_{n} C \leq_{n} B$. Then, there exist $A^{\prime}, B^{\prime} \in\left(I^{\prime}\right)^{*}$ such that $A=\phi^{-1}\left(A^{\prime}\right), B=$ $\phi^{-1}\left(B^{\prime}\right)$. Now, $\phi^{-1}\left(A^{\prime}\right) \leq_{n} C \leq_{n} \phi^{-1}\left(B^{\prime}\right)$, implies $\phi\left(\phi^{-1}\left(A^{\prime}\right)\right) \leq_{n} \phi(C) \leq_{n}$ $\phi\left(\phi^{-1}\left(B^{\prime}\right)\right)$, by Lemma 4.11. That is, $A^{\prime} \leq_{n} \phi(C) \leq_{n} B^{\prime}$. Since $\left(I^{\prime}\right)^{*}$ is convex and $\phi(C) \in M_{n}\left(N^{\prime}\right)$, we get $\phi(C) \in\left(I^{\prime}\right)^{*}$, which implies $C \in \phi^{-1}\left(I^{\prime}\right)^{*}=I^{*}$. Therefore, $I^{*}$ is convex in $M_{n}(N)$. Next we show that $M_{n}(N) / I^{*} \simeq_{0} M_{n}\left(N^{\prime}\right) /\left(I^{\prime}\right)^{*}$. Since $I$ is an ideal of $N$, by Lemma $4.14, M_{n}(N / I) \simeq M_{n}(N) / I^{*}$, similarly, if $I^{\prime}$ is an ideal of $N^{\prime}$, then $M_{n}\left(N^{\prime} / I^{\prime}\right) \simeq M_{n}\left(N^{\prime}\right) /\left(I^{\prime}\right)^{*}$. Again since, $N / I \backsim \simeq_{0} N^{\prime} / I^{\prime}$, by Theorem 4.11 , it follows that $M_{n}(N / I) \simeq 。 M_{n}\left(N^{\prime} / I^{\prime}\right)$. Therefore, $M_{n}(N) / I^{*} \simeq_{\circ} M_{n}\left(N^{\prime}\right) /\left(I^{\prime}\right)^{*}$.

\section{Archimedean Order in Matrix Nearring}

Definition 5.1 An ordered matrix nearing $M_{n}(N)$ is called Archimedean if $\left(M_{n}\right.$ $(N),+)$ is Archimedean. That is, for any $A, B \in \mathcal{P}, k A \leq_{n} B$ for all $k \in \mathbb{Z}^{+}$, implies $A=\overline{\mathbf{0}}$.

Corollary 5.2 [11] $N$ is abelian iff $M_{n}(N)$ is abelian.

Proposition 5.3 If $N$ isf.o. Archimedean, then $M_{n}(N)$ is f.o. Archimedean and $M_{n}(N)$ is abelian.

Proof Suppose $N$ is f.o. Archimedean. First, we show that $(N,+)$ is abelian.

Case (i): Let $a \in N$ such that $a>0$ and $0 \leq x<a$ implies $x=0 \cdots(*)$, say. We have for each $b \in N$, there exist $n \in \mathbb{Z}^{+}$such that $n a \leq b<(n+1) a$. This implies that $0 \leq-n a+b<a$. Now, by assumption $(*)$, we have $-n a+b=0, b=n a$.

Case (ii): Assume that no such ' $a$ ' exists as in Case (i). For every $x \in N, 0<x$, we find $y \in N$ such that $0<y<x$. Suppose that $2(x-y) \not \leq x$. Since $N$ is f.o., $2(x-y) \geq x$. That is, $(x-y)+(x-y) \geq x$, implies that $-x+x-y+x-y \geq 0$. Therefore, $-y+x \geq y$, and so $x \geq 2 y$. Hence, $2 y \leq x$ or $2(x-y) \leq x$. Thus, to each $x>0$, there exists $z \in N$ such that $0<z<x$ and $2 z \leq x$. Assume that $a, b \in N^{+}$ with $a+b \neq b+a$. Suppose $a+b>b+a$. Put $x=a+b-a-b>0$. Let $z \in N$ 
such that $0<z<x$ and $2 z \leq x$. Since $N$ is Archimedean, there exists $m, n \in \mathbb{Z}^{+}$ satisfying $m z \leq a<(m+1) z$ and $n z \leq b<(n+1) z$. This implies that

$$
m z+n z \leq a+b<(m+1) z+(n+1) z \cdots(1) .
$$

In addition, $-(m+1) z<-a \leq-m z$ and $-(n+1) z<-b \leq-n z$, imply that

$$
-(m+1) z-(n+1) z<-a-b \leq-m z-n z \cdots(2)
$$

Adding (1) and (2) we get,

$$
m z+n z-(m+1) z-(n+1) z<x<(m+1) z+(n+1) z-m z-n z,
$$

implies that

$$
[m+n-(m+1)-(n+1)] z<x<[(m+1)+(n+1)-m-n] z .
$$

Therefore, $-2 z<x<2 z$, hence, $x<2 z$, a contradiction to $2 z \leq x$. Therefore, $a+b=b+a$. Hence $(N,+)$ is abelian. Now, by Corollary 5.2, it follows that $\left(M_{n}(N),+\right)$ is abelian. Next we prove that $M_{n}(N)$ is Archimedean ordered. Suppose $\overline{\mathbf{0}} \leq_{n} A, \overline{\mathbf{0}} \leq_{n} B, k A \leq_{n} B$ for all $k \in \mathbb{Z}^{+}$. To show $A=\overline{\mathbf{0}}$, let $A=f_{i j}^{r}$ and $B=f_{i j}^{s}$. Now, $k f_{i j}^{r} \leq_{n} f_{i j}^{s}$, implies that $\pi_{i}\left(k f_{i j}^{r} \rho\right) \leq \pi_{i}\left(f_{i j}^{s} \rho\right)$, for all $\rho \in\left(N^{+}\right)^{n}, 1 \leq i \leq n$.

That is,

$$
\pi_{i}(\underbrace{\left(f_{i j}^{r}+f_{i j}^{r}+\cdots+f_{i j}^{r}\right)}_{k \text { times }} \rho) \leq \pi_{i}\left(f_{i j}^{s} \rho\right), \text { for all } \rho \in\left(N^{+}\right)^{n} .
$$

In particular,

$$
\pi_{i}\left(f_{i j}^{k r}\langle 1,1, \ldots, 1\rangle\right) \leq \pi_{i}\left(f_{i j}^{s}\langle 1,1, \ldots, 1\rangle\right)
$$

Therefore, $\pi_{i}\left(\langle 0,0, \ldots, \underbrace{k r}_{i^{t h}}, \ldots, 0\rangle\right) \leq \pi_{i}\left(\langle 0,0, \ldots, \underbrace{s}_{\mathrm{i}^{\text {th }}}, \ldots, 0\rangle\right)$, and hence, $k r \leq s$ for all $k \in \mathbb{Z}^{+}$. Since $N$ is Archimedean, we get $r=0$. Now,

$$
\begin{aligned}
f_{i j}^{r}\left\langle a_{1}, a_{2}, \ldots, a_{n}\right\rangle & =\langle 0,0, \ldots, \underbrace{r a_{j}}_{i^{t h}}, \ldots, 0\rangle \\
& =\langle 0,0, \ldots, \underbrace{0 a_{j}}_{i^{t h}}, \ldots, 0\rangle \\
& =\langle 0,0, \ldots, 0\rangle=f_{i j}^{0}\left\langle a_{1}, a_{2}, \ldots, a_{n}\right\rangle
\end{aligned}
$$

for all $\left\langle a_{1}, a_{2}, \ldots, a_{n}\right\rangle \in N^{n}$. Hence, $f_{i j}^{r}=\overline{\mathbf{0}}$. Therefore, $M_{n}(N)$ is Archimedean. 
The following definition is analogous to ([10], Definition 9.140).

Definition 5.4 If $M_{n}(N)$ is not Archimedean, then for any $A, B \in M_{n}(N), A$ is small with respect to $B$, if

$$
k|A|=\underbrace{(|A|+|A|+\cdots+|A|)}_{\mathrm{k} \text { times }}<_{n}|B|, \text { for all } k \in \mathbb{Z}^{+},
$$

where $|A|=\max (A,-A)$. That is, $A \leq_{n}-A$ or $-A \leq_{n} A$.

Definition $5.5 A \in M_{n}(N)$ is nearly constant if and only if $A_{0} \ll_{n}\left[\left(M_{n}(N)\right)_{c}\right]^{*}$.

Lemma 5.6 [11] $r$ is constant in $N$ iff $f_{i j}^{r}$ is constant in $\mathbb{M}_{n}(N)$.

Proposition 5.7 Let $N$ be f.o. and $N_{c} \neq\{0\}$. If $r \in N$ is nearly constant in $N$, then $f_{i j}^{r}$ is nearly constant in $M_{n}(N)$.

Proof Suppose $r \in N$ is nearly constant. Then, $r_{0} \ll N_{c}^{*}$ and $k\left|r_{0}\right|<b$ for all $b \in N_{c}^{*}$. Now, $\underbrace{\left(\left|r_{0}\right|+\left|r_{0}\right|+\cdots+\left|r_{0}\right|\right)}_{\mathrm{k} \text { times }}<b$ implies that $k r_{0}<b$ or $-k r_{0}<b$.

Suppose $k r_{0}<b$. To show $f_{i j}^{k r_{0}}<_{n} f_{i j}^{b}$, let $\rho=\left\langle a_{1}, a_{2}, \ldots, a_{n}\right\rangle \in N^{n}$. Now,

$$
\begin{aligned}
\pi_{i}\left(f_{i j}^{k r_{0}} \rho\right) & =f_{i j}^{k r_{0}}\left\langle a_{1}, a_{2}, \ldots, a_{n}\right\rangle \\
& =\pi_{i}\left(\langle 0,0, \ldots, \underbrace{k r_{0} a_{j}}_{\mathrm{i}^{\text {th }}}, \ldots, 0\rangle\right) \\
& \leq \pi_{i}\left(\langle 0,0, \ldots, \underbrace{b a_{j}}_{\mathrm{i}^{\text {th }}}, \ldots, 0\rangle\right) \\
& =\pi_{i}\left(f_{i j}^{b}\left\langle a_{1}, a_{2}, \ldots, a_{n}\right\rangle\right)=\pi_{i}\left(f_{i j}^{b} \rho\right) .
\end{aligned}
$$

Therefore, $f_{i j}^{k r_{0}}<_{n} f_{i j}^{b}$. In a similar way, we get $f_{i j}^{-k r_{0}}<_{n} f_{i j}^{b}$. Now, $f_{i j}^{k r_{0}}<_{n} f_{i j}^{b}$ or $f_{i j}^{-k r_{0}}<_{n} f_{i j}^{b}$, it follows that $f_{i j}^{k\left|r_{0}\right|}<_{n} f_{i j}^{b}$. Now, we show $f_{i j}^{b} \in\left[\left(M_{n}(N)\right)_{c}\right]^{*}$. Since $b$ is constant in $N$, by Lemma 5.6, $f_{i j}^{b}$ is constant in $M_{n}(N)$. Since $b \neq 0$, if $f_{i j}^{b}=\overline{0}$, then $f_{i j}^{b}\left\langle a_{1}, a_{2}, \ldots, a_{n}\right\rangle=\overline{0}$ for all $\left\langle a_{1}, a_{2}, \ldots, a_{n}\right\rangle \in N^{n}$. Now, $\langle 0,0, \ldots, 0\rangle=$ $f_{i j}^{b}\langle 1,1, \ldots, 1\rangle=\langle 0,0, \ldots, \underbrace{b}_{i^{t h}}, \ldots, 0\rangle$, implies $b=0$, a contradiction. Therefore,

$f_{i j}^{b} \in\left[\left(M_{n}(N)\right)_{c}\right]^{*}$, implies $f_{i j}^{k\left|r_{0}\right|}<_{n} f_{i j}^{b}$ for all $f_{i j}^{b} \in\left[\left(M_{n}(N)\right)_{c}\right]^{*}$. Hence, $f_{i j}^{r}$ is nearly constant in $M_{n}(N)$.

\section{Conclusion}

There are enormous developments in the study of partial order in algebraic systems such as groups, rings, and modules. In this paper, we have extended the notion of partial order to the matrix nearrings wherein its base nearring is partially ordered with 
identity. Convexity is one of the significant properties in p.o. nearrings; accordingly, we have defined the convex ideal of a matrix nearring corresponding to a convex ideal in a p.o. nearring. We have established some properties and isomorphism theorems of convex ideals of p.o. matrix nearrings. One can extend the study to the ideal theory of lattice ordered concepts in matrix nearrings corresponding to the existing ideal theory of lattice ordered nearrings.

Acknowledgements The authors express their deep gratitude to the referee(s)/editor(s) for their careful reading of the manuscript, and valuable suggestions that have definitely improved the paper. S. Tapatee, P.K. Harikrishnan, B.S. Kedukodi, S.P. Kuncham acknowledge Manipal Institute of Technology (MIT), Manipal Academy of Higher Education, Manipal, India for their kind encouragement. J. H. Meyer acknowledges the University of the Free State, Bloemfontein, South Africa.

Funding Open access funding provided by Manipal Academy of Higher Education, Manipal.

Open Access This article is licensed under a Creative Commons Attribution 4.0 International License, which permits use, sharing, adaptation, distribution and reproduction in any medium or format, as long as you give appropriate credit to the original author(s) and the source, provide a link to the Creative Commons licence, and indicate if changes were made. The images or other third party material in this article are included in the article's Creative Commons licence, unless indicated otherwise in a credit line to the material. If material is not included in the article's Creative Commons licence and your intended use is not permitted by statutory regulation or exceeds the permitted use, you will need to obtain permission directly from the copyright holder. To view a copy of this licence, visit http://creativecommons.org/licenses/by/4.0/.

\section{References}

1. Booth, G.L., Groenewald, N.J.: On primeness in matrix near-rings. Archiv der Mathematik 56(6), 539-546 (1991)

2. Bhavanari, S., Kuncham, S.P.: Nearrings, Fuzzy Ideals, and Graph Theory. CRC Press, London (2013)

3. Bhavanari, S., Lokeswara Rao, M.B.V., Kuncham, S.P.: A note on primeness in near-rings and matrix near-rings. Indian J. Pure appl. Math. 27, 227-234 (1996)

4. Bhavanari, S., Kuncham, S.P.: On finite Goldie dimension of $M_{n}(N)$-group $N^{n}$. In: Proceedings of the Conference on Nearrings and Nearfields, pp. 301-310. Springer (2005)

5. Fuchs, L.: Partially Ordered Algebraic Systems, p. 28. Dover Publ., Inc., New York (2011)

6. Jingjing, M.: Lecture Notes on Algebraic Structure of Lattice-ordered Rings. World Scientific, Singapore (2014)

7. Ke, W. F.: Planar nearrings: Ten ten years after. In: Kuncham, S.P., Kedukodi, B.S., Harikrishnan, P.K., Bhavanari, S., Neuerburg, K., Booth, G.L., Davvaz, B., Farag, M., Juglal, S. and Badawi, A. (eds.) Nearrings, nearfields and related topics. World Scientific, Singapore (2017)

8. Kuncham, S.P., Kedukodi, B.S., Harikrishnan, P.K., Bhavanari, S. (eds.): Nearrings, Nearfields and Related Topics (Review Volume). World Scientific Publ. Co., Singapore (2017)

9. Pilz, G.: On direct sums of ordered nearrings. J. Algebra 18, 340-342 (1971)

10. Pilz, G.: Near-Rings: the Theory and its Applications, p. 23. North Holland, Amsterdam (1983)

11. Meldrum, J.D.P., Van der Walt, A.P.J.: Matrix near-rings. Archiv der Mathematik 47(4), 312-319 (1986)

12. Meldrum, J.D.P., Meyer, J.H.: Intermediate ideals in matrix near-rings. Commun. Algebra 24(5), 16011619 (1996)

13. Meyer, J.H.: Left ideals in matrix near-rings. Commun. Algebra 17(6), 1315-1335 (1989)

14. Meyer, J.H.: Introduction to Matrix Nearrings, Review Volume Entitled, Nearrings, Nearfields and Related Topics, pp. 152-158. World scientific, Singapore (2017)

15. Meyer, J.H.: Left ideals and 0-primitivity in matrix near-rings. Proc. Edinb. Math. Soc. 35(2), 173-187 (1992) 
16. Meyer, J.H., On the Development of matrix nearrings and related nearrings over the past decade. In: Near-rings and Near-fields, pp. 23-34. Springer, Dordrecht (2001). https://doi.org/10.1007/978-94010-0954-6_3

17. Radhakrishna, A.: On lattice ordered nearrings and non-associative rings. Doctoral thesis, submitted to IIT Kanpur, India (1975)

18. Radhakrishna, A., Bhandari, M.C.: On a class of lattice ordered nearrings. Indian J. Pure Appl. Math. 9(6), 581-587 (1977)

19. Steinberg, S.A.: Lattice-Ordered Rings and Modules. Springer, New York (2010)

20. Van Der Walt, A.P.J.: Primitivity in matrix near-rings. Quaestiones Mathematicae 9(1-4), 459-469 (1986)

21. Van Der Walt, A.P.J.: On two-sided ideals in matrix near-rings. N.-Holl. Math. Stud. 137, 267-271 (1987)

22. Zayed, M.: On matrix near-rings. Archiv der Mathematik 77(2), 163-169 (2001)

Publisher's Note Springer Nature remains neutral with regard to jurisdictional claims in published maps and institutional affiliations. 